\title{
Does resistance of Anopheles mosquitoes to knock-out effect of DDT deter the ability of the chemical to control malaria in Uganda?
}

\author{
G. S. BIMENYA *, P. S. MUGISHA, A. L. OKWI, M. HABARULEMA and \\ M. LUGEMWA
}
Pathology Department, School of Biomedical Sciences, College of Health Sciences, Makerere University P. O. Box 7072, Kampala, Uganda.
*Corresponding author,E-mail: gsbimenya@chs.mak.ac.ug

\begin{abstract}
The WHO/HQ, Geneva, developed general guidelines for monitoring and management of insecticide resistance. These were adapted for local conditions by the WHO/AFRO and were made available to national programs for use. The guidelines proposed strategies to prevent/delay development of resistance; approaches to minimize impact of resistance should it appear; and possible alternative strategies in case of resistance reached to a level requiring replacement of the insecticides in use. For Africa South of the Sahara, at least six organizations formed a partnership called the African Network on Vector Resistance to insecticides (ANVR). This network made sub-partnerships with national research institutions, national vector control programs and the private sector in each African country to make large-scale entomological surveys focusing on vector susceptibility to insecticides and updating vector distribution throughout the WHO African region. In Uganda, ANVR made their appropriate studies and established that the major malaria vector, Anopheles gambiae, was resistant to the vector control chemicals in use (DDT and Icon). They showed that new chemicals, the carbamates and organophosphates, were fully efficacious on the local vector and recommended immediate change to the new chemicals. Commissioned by the Ministry of Health $(\mathrm{MoH})$, this work followed up the ANVR experiments in Uganda, compared the issues accrued with those on record from similar studies done in Kihihi in 1957-1960 and found that the ANVR studies were well designed and well done but the interpretation of the results only favoured their objectives.

(c) 2010 International Formulae Group. All rights reserved.
\end{abstract}

Keywords: Insecticide, repellancy, vector, irritance, IRS, susceptibility.

\section{INTRODUCTION}

From time immemorial, malaria caused havoc to humanity on kings and commoners alike and decimated settlements and civilizations until effective control of the vector mosquito and affordable treatment of the patients were concurrently carried out in the affected regions. In southern Europe, eradication of malaria transmission was achieved by spraying of dichlorodiphenyltrichloroethane (DDT) in 1940s and 1950s sustained by adequate treatment of malaria cases (Curtis, 2002). After the World War II, between 1947 and 1952, the USA eradicated malaria from its territory by fighting mosquitoes with DDT and by treating malaria with chloroquine (Pautian, 2000). In 1955 the WHO declared an 
Eight-year Global Malaria Eradication Campaign using DDT but in Africa these attempts were made in only three countries (Offobuche, 2008) which included Uganda.

In Uganda, Malaria Eradication Pilot Project was carried out between 1957 and 1960 by studying mosquito behavior under indoor residual spraying (IRS) with DDT and mass administration of antimalarial drug tablets containing chloroquine (200 $\mathrm{mg})$ and pyremethamine $(16.5 \mathrm{mg})$ in the malariahyper-endemic, non-habitable Rwangaminyeto area of Butumbi region of North Kigezi, currently the Kihihi sub-county of Kanungu District. The project was so successful that the researchers reported to $\mathrm{WHO}$ that the results so far obtained in Kigezi indicate the possibility of malaria eradication in the area within a short time. Preliminary surveys in other parts of western and central Uganda indicate that no more malaria is to be found there than in the north of Kigezi, thus showing that the eradication of malaria in central and western Uganda is feasible (De Zulueta et al., 1961). Kihihi is now a flourishingly cosmopolitan sub county with an airfield soon to be promoted to an international airport, due to tourism and trade.

The Eight-year Global Plan of WHO to eradicate malaria using, inter alia, DDT was interrupted by many forces, particularly the following three:

(1) The quest for independence by the African territories during the 1960s: Uganda got independent in 1962 and malaria ceased to be a priority to the colonial or the emerging government. In any case, the new governments lacked the science, the infrastructure and the logistics effectively to carry out the eradication campaigns;

(2) The Silent Spring, a book written by a science journalist, Rachel Carson and published in 1962 incessantly criticizing the use of DDT for agricultural purposes and spelling doom for the use of the chemical for any thing; this activated the environmentalism which factored in with the $3^{\text {rd }}$ force, hereunder (Offoboche, 2008);

(3) The Malthusians who were uncomfortable with the growing population of the developing countries, especially in Africa (Offoboche, 2008) saw malaria as a control agent.

The combined effects of the three forces eased the fight against using DDT for malaria control. It created unprecedented menticide of generations against the use of DDT todate (Mazel, 2002). In Africa malaria soared, the continent wailed as it buried its dead from malaria everyday and subsequently three seemingly sympathetic initiatives evolved: The Roll Back Malaria (RBM), the Global Fund to fight AIDS, Tuberculosis and Malaria (GFATM) and the Bill and Melinda Gates Fund.

As the funds were availed seemingly to fight malaria in Africa, three things related to malaria happened in Uganda:

a) Insecticide-coated mosquito nets were ushered in aggressively:

These mosquito nets may be very useful to tots who sleep under them and wet their beds, but they are not so useful to adults especially pregnant women and men who take evening beverages. No wonder, the measure of their success was not on the parasite index but the reduction in child mortality rate (Offoboche, 2008);

b) Chloroquine was declared ineffective for malaria treatment. Before chloroquine was deshelved, an adult dose of 10 tablets used to cost $\mathrm{Ug}$ Shs 500 (about US\$0.26). This gave way to Ug Shs 20,000 (US \$10.53) for one adult dose of artemesin combination on open market. This meant a perpetually high expenditure for health in both the national and personal budgets and chloroquine used along DDT in eradication/ elimination/control of malaria was no longer available. Half of the effective weaponry against malaria was lost 
while the other half, DDT, was severely restricted by Stolkholm convention (UNEP/POPS/DDTBP.1/2, 2008);

c) Malaria consortia were formed: the effect of this will emerge from the discussion concerning in door residual spray (IRS) chemicals.

Since June, 2006 IRS of DDT was officially allowed under the watch of WHO, but it seems malaria will not be eradicated from Africa till WHO addresses the "Malthusian" green opposition' (Offoboche, 2008). In 2008 Uganda became one of the ten African countries to use DDT in malaria control through IRS. The IRS effort started with the worst-hit by malaria northern-district of Apach (Bimenya et al., 2009) (Figure 1). At the dawn of the $21^{\text {st }}$ century, the world "anti" malaria forces formed alliances known by various names including. "The African Network on Vector Resistance to Insecticides" (ANVR).

The network comprising of at least six influential partnerships works throughout the WHO Black African Region to generate sound, evidence-based management interventions to establish malaria vector resistance and to prevent its emergency where the resistance doesn't already exist. Uganda: Tororo, Apac, Wakiso, Hoima and Kanungu districts were studied by ANVR. Their locations were as shown in Figure 1.

Typical results of ANVR 2000-2009 studies in these districts were as shown in Table 1.

It was concluded that the Anopheles mosquitoes were resistant to DDT and LCT in all the districts, the worst being Kanungu District where DDT IRS had been done in 1957-1960 studies and a pyrethroid was applied in 2006. In all the districts, the mosquitoes were fully susceptible to carbamates and the organophosphates.

It was then recommended that a change be made immediately from using pyrethroids and DDT to either carbamates (e.g. BDC) or to organophosphates (e.g. PPM). Also it was recommended that the insecticides be used in a mosaic manner to avoid resistance development.

It is against the above background that this study compares the field research results, conclusions and recommendations of the ANVR's Malaria Vector Susceptibility to Public Health Insecticides in Uganda (20002009) with those from the first year of malaria eradication pilot project in northern Kigezi (Uganda) in 1957-1960 (De Zulueta et al., 1961) when the WHO still had the eradication program. The aim is to show the essence of bioassay tests in prescribing DDT for malaria eradication, elimination or control as was done by the 1957-1960 studies or proscribing it against the same as was done in the 20002009 studies.

\section{MATERIALS AND METHODS}

Data used in this work were gleaned from different sources as follows:

a) From our own data on experiments done at the ANVR centres in Uganda with official protocol. The Anopheles susceptibility tests were done as shown in Figure 2.

Plastic tubes fitted at one end with a mesh screen and marked with a red dot are each lined with a filter paper impregnated with the test chemical and a hundred mosquitoes were gently put into the tubes for exposure to the chemical for sixty minutes. For each test chemical, a similar tube lined with a plain filter paper and marked with a green dot hosted a hundred mosquitoes as controls.

b) The 1957-1960 data retrieved from the Ugandan entomologists who participated in the campaign and are still alive, local government records where applicable, the ministry of health $(\mathrm{MoH})$ of Uganda and from WHO publications.

c) The 2000-2009 data obtained from ANVR results in Uganda submitted to the MoH.

d) The bioassay data from abroad in literature. Both sets of main researchers of 19571960 and 2000-2009 in Uganda, inter alia, captured malaria vectors in houses, got larva from breeding places and nurtured them in laboratory insectaries for various experiments. 
Our concerns are the bioassays on the most important adult malaria vector in Uganda, the Anopheles gambiae. In 1957-1960 the field research conditions in Kihihi were: female mosquitoes from non sprayed experimental houses tested in the fed state on $4 \%$ technical grade DDT prepared locally on standard filter papers exposed to the mosquitoes for 30 minutes, with limited control on temperature, humidity, atmospheric pressure and lighting (De Zulueta et al., 1961) whereas the 20002009 conditions were wild female mosquitoes tested in the fed state under well controlled conditions of temperature, humidity, and lighting, on filter papers of 4\% DDT imported already prepared through $\mathrm{WHO}$ and exposed to the mosquitoes for 60 minutes (PMI/USAID/MOH/UG/Malaria consortium protocol).

Knock-down assays were done in Kihihi both in 1959 and 2009 studies. Knock down bioassays were tests in which a given number of mosquitoes under standard conditions were subjected to chemicals according to WHO protocol and the number of mosquitoes that died in a given time was recorded.

Susceptibility tests were done on mosquitoes both in 1959 and 2009 in Kihihi. Whereas in Kihihi in 1957-1960 only technical grade DDT was studied, the susceptibility tests in 2000-2009 involved ten chemicals generally arranged in the following order: DDT 4\%, Lambdacyhalothrin $0.05 \%$, Bendiocarb $0.1 \%$, Pirimophos-methyl $1 \%$, Deltamethrin $0.05 \%$, Permethrin $0.75 \%$, Propoxur $0.1 \%$, Malathion 5\%, Cyfluthrin $0.15 \%$, Etofenprox $0.5 \%$ (ANVR UG protocol). After exposure of the mosquitoes to the test chemicals, the mosquitoes are gently transferred into holding tubes for fate observation as shown in Figure 3.

The methods, results, conclusions and recommendations mainly of the De Zulueta et al. (1961) and the ANVR related group studies (2000-2009) in Uganda and reported to $\mathrm{MoH}$ were compared as the main research studies and discussed along related bioassays from us and abroad. A serious point of contention is the recommendation of the 1957-1960 studies upheld by WHO todate that substances for in-door residual spray must be studied in each area for their availability, cost, residual effectiveness, safety, vector susceptibility, irritability, toxicity and excitorepellency (WHO, 2006.3; DeZulueta et al., 1961; Cullen and De Zulueta, 1962).

\section{RESULTS}

\section{Results of knock-down bioassays with DDT}

Table 2 shows the results from the same Kihihi subcounty in both 1957-1960 and 2000-2009 studies.

Source: 1959 results were adapted from Table XII of De Zulueta et al. (1961), whereas the 2009 results were adopted from ANVR 2000-2009 study report to $\mathrm{MoH}$.

Although these results are both retrospective and not a replica of each other, they still show that DDT is not a quick killer of Anopheles mosquitoes. In fact DDT can hardly be called a killer of Anopheles gambiae at all, because even within 24 hours, only less than $20 \%$ of the insects were killed in both 1957-1960 and 2000-2009 studies. This means that in Uganda, the knock-down effect of DDT on Anopheles gambiae mosquitoes in 2009 is as low as it was in 1957-1960.

\section{Susceptibility results}

Table 3 shows our susceptability results of Anopheles gambiae mosquitoes to four of those chemicals in ANVR field station obtained on 22nd Oct. 2009.

The results obtained were typical of the 2000-2009 studies on MoH records.

a) DDT (organochlorine), BDC (Carbamate), PPM (organophosphate), PPX (Carbamate).

b) Since the test sample is $n=100$, the number of mosquitoes dead in $24 \mathrm{~h}$ is equivalent to the mortality percentage expressed in Table 1.

These results obtained by the authors in the field on $22^{\text {nd }}$ October 2009 using the ANVR (Uganda) facilities and protocols show that DDT does not kill as fast nor as much as the other chemicals. For knock-down 
purposes, the carbamate (BDC) and the organophosphate (PPX) excel.

\section{0-2009 irritability studies in Uganda}

In 2000-2009, no overt irritability studies, were done and therefore there are no records. However, from the susceptibility experiments it could be observed that in the exposure stage (Figure 2), with DDT and LCT, the mosquito behaviour in the exposure and in post exposure holding tubes (Figure 3) changed thus:

a) The mosquitoes scampered to the top of the tube in attempt to escape, but the tube netting stopped them; there was no scampering in the control tubes.

b) The mosquitoes were agitated making loudly hostile noise, very different from the soothing tonality made when attempting to bite; the control tubes were serene.

c) The mosquitoes crowded to the top of the tube as in search of fresh air and light; the controls remained in the tubes.

d) Where room was available the mosquitoes curled their hind legs as if to avoid padding with the toxicant/irritant; the controls rested normally.

These were typical signs of repellency and irritability (Sollers-Riedel, 1963), which were conveniently not considered by the 2000-2009 studies.

Table 4 shows one of the experimental results, illuming the irritability of DDT on the mosquitoes, leading them to go into exile from the sprayed quarters in 1959 at Kihihi and Katojo.

It showed that the mosquitoes were highly irritated by the DDT and attempted many times to escape but were confined in the test containers by the wire mesh. This test formerly used to study both the irritability and repellence was retained for irritability. Repellence tests need better experimental designs such as those of Potikaskorn et al. (2005) whose part results are shown in this paper.

\section{Studies from abroad}

Following the studies done in Kihihi, recommendations were made concerning the study designs on alteration of behaviour of mosquito vectors by chemicals intended for vector control: irritability, excito/repellence, space/contact toxicity as in Table 5.

a) $\mathrm{DDT}=\mathrm{DDT} 2 \mathrm{~g} / \mathrm{m}^{2}$ on filter paper using WHO protocol.

b) LCT=Lambddacyahalothin $0.03 \mathrm{~g} / \mathrm{m}^{2}$ on filter paper using WHO protocol.

c) Excito-repellence chamber allowing the choice between staying and escaping was used as shown in Figure 4.

These results show that in a modern equipment where the vectors have a choice to flee or to stay:

1. DDT and LCT exile most of the Anopheles vectors, DDT at $92 \%$ and LCT at $94 \%$ rates.

2. The repellence effects of DDT and LCT on Anopheles species are approximately equal.

3. The repellence effects for both chemicals are equal for contact and non contact modes.

4. When the mosquitoes are exiled by contact irritation, they eventually die from contact toxicity and this effect is stronger in LCT than with DDT.

5. With both DDT and LCT, non contact repellence does not necessarily kill the mosquitoes.

6. Both DDT and LCT act as primarily repellants, secondarily irritants and lastly as not so strong knock out insecticides.

These six properties of DDT and LCT seen in the exposure (Figure 2) and post exposure holding tubes (Figure 3), buttressed in their residual longevity and relatively low price seem to have them considered impotent in malaria vector control in Uganda today. These properties which were intensively studied in 1957-1960 and are recommended by WHO for every region (WHO, 2006.3) were omitted conveniently in the 2000-2009 studies. 


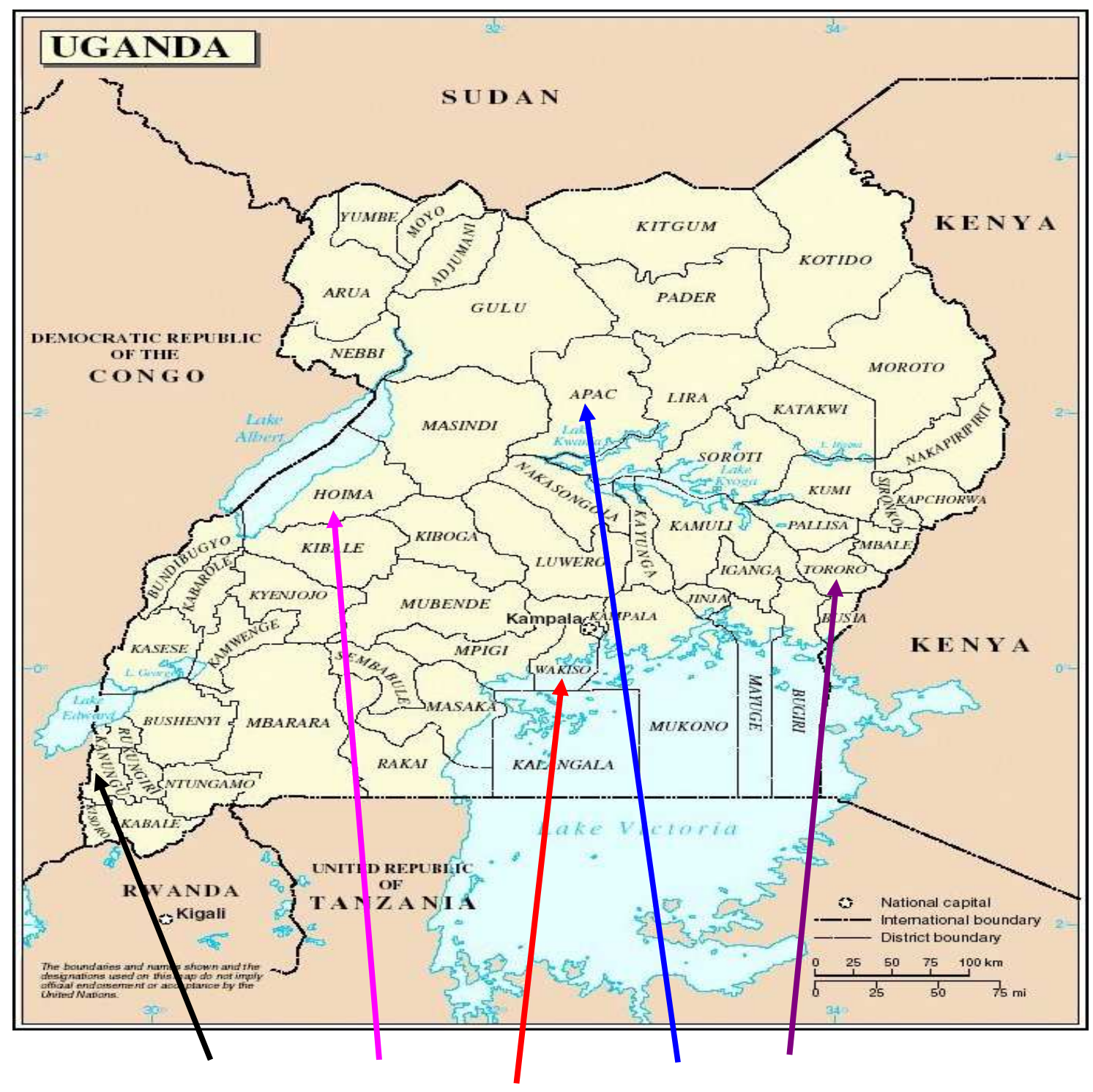

Figure 1: Representative Districts involved in vector resistance studies 2000-2009.

Table 1: The 2000-2009 ANVR susceptibility results reported to MoH, Uganda.

\begin{tabular}{cccccc}
\hline Chemical & $\begin{array}{c}\text { Wakiso } \\
\text { District }\end{array}$ & $\begin{array}{c}\text { Apac } \\
\text { District }\end{array}$ & $\begin{array}{c}\text { Hoima } \\
\text { District }\end{array}$ & $\begin{array}{c}\text { Kanungu } \\
\text { District }\end{array}$ & $\begin{array}{c}\text { Tororo } \\
\text { District }\end{array}$ \\
\hline DDT & 33 & 52 & 52 & 16 & 48 \\
BDC & 100 & 100 & 100 & 100 & 100 \\
PPM & 100 & 100 & 100 & 100 & 100 \\
LCT| & 95 & 68 & 89 & 27 & 75 \\
\hline DT (dichlorodiphenyltrichloethane, an organochlorine), BDC (Bendiocarb, & a carbamate), & PPM \\
(Pirimiphosphomethyl, an organophosphate), LCT (a pyrethroid Lambdacyhalothrin). &
\end{tabular}




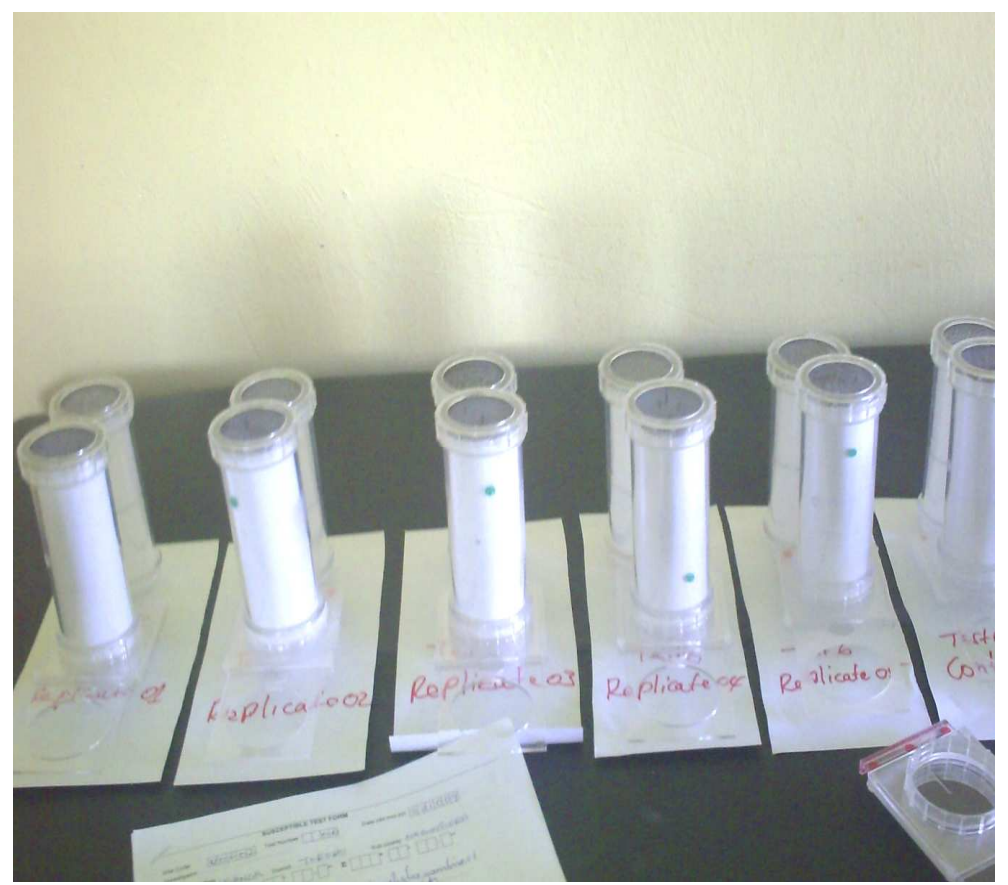

Figure 2: The susceptibility/vector resistance study tubes of our work on $21^{\text {st }}$ Oct 2009.

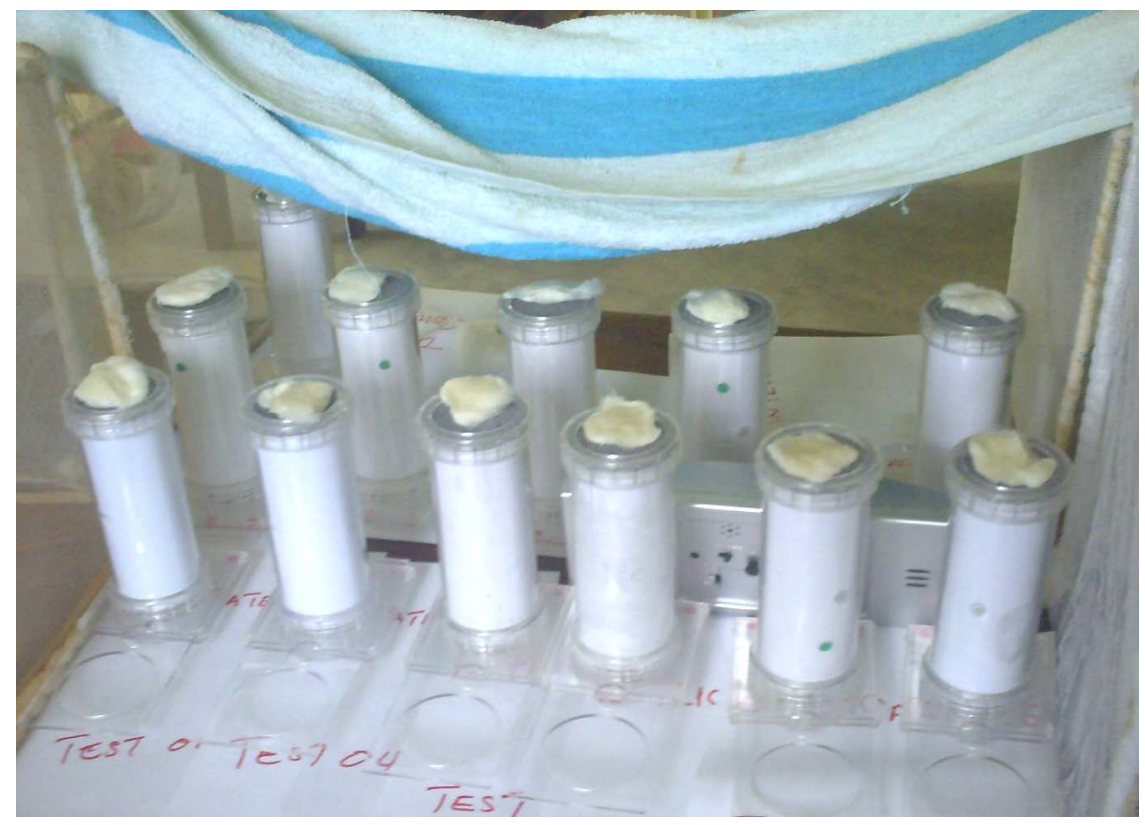

Figure 3: Our 24-hour holding process on $21^{\text {st }}-22^{\text {nd }}$ October 2009.

a) Conditions of temperature, lighting, humidity etc are held approximately constant

b) The cotton wool on top of the wire mesh is soaked in mosquito feed solution

c) After 24 hour hold, the mosquito mortality was determined in both the test and its control tubes for each chemical

d) According to the test protocol mortality rates equal to or above $20 \%$ in the controls make the study repeated. 


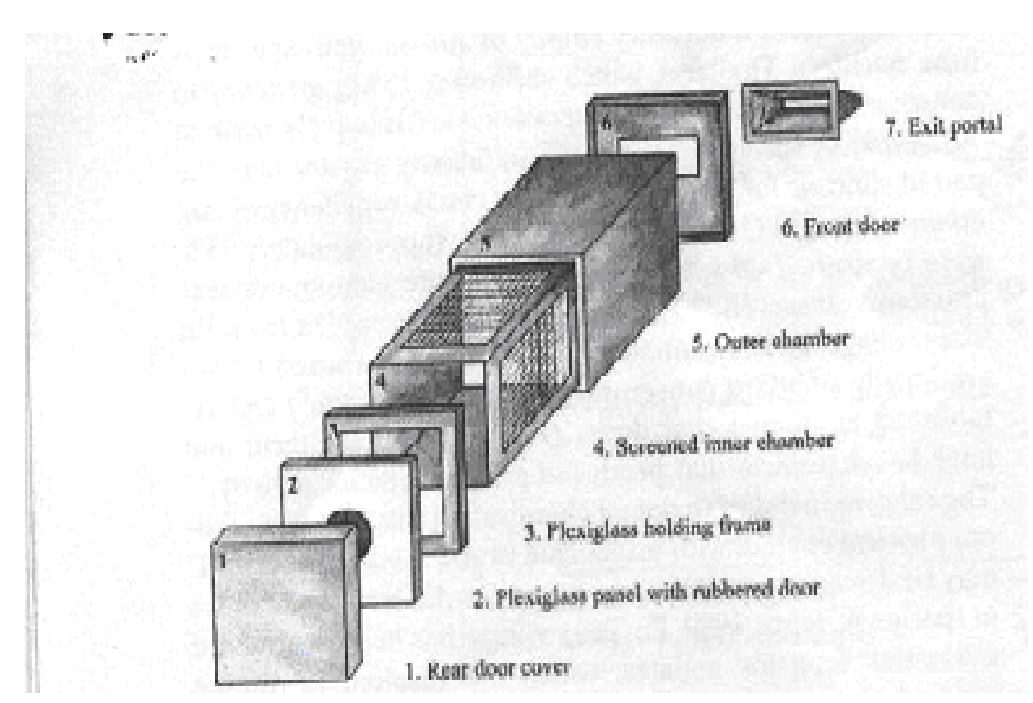

Figure 4: A modern excito-repellency test chamber for mosquito behaviour studies. Source: Adopted from Potikaskorri et al., 2005.

Table 2: Results of knock-down bioassays with DDT on Kihihi mosquitoes.

\begin{tabular}{lccc}
\hline Date & No. of tested mosquitoes & No. of dead mosquitoes in 24 hours & Mortality \\
Sept. 1959 & 40 & 7 & 18 \\
Sept. 2009 & 100 & 13 & 13 \\
\hline
\end{tabular}

Table 3: Our 2009 susceptibility results in the field using ANVR Uganda protocol.

\begin{tabular}{lcccc}
\hline Chemical & No. of tested mosquitoes & $\begin{array}{c}\text { No. Dead in } \\
\mathbf{2 4} \text { hours }\end{array}$ & 1hr Dead \% & $\begin{array}{c}\text { Minutes of } \\
\text { n/2 Demise }\end{array}$ \\
\hline DDT & 100 & 54 & 30 & $>60$ \\
BDC & 100 & 100 & 95 & 40 \\
PPM & 100 & 87 & 93 & 30 \\
PPX & 100 & 100 & 99 & 30 \\
\hline
\end{tabular}

Table 4: Number of mosquito-take offs.

\begin{tabular}{|c|c|c|c|c|c|c|}
\hline \multirow[t]{2}{*}{ Species } & \multirow{2}{*}{ Chemical } & \multirow{2}{*}{$\begin{array}{l}\text { Number } \\
\text { exposed }\end{array}$} & \multicolumn{4}{|c|}{ Number of take offs in each period } \\
\hline & & & 1 min & 5 min & 10 min & $15 \mathrm{~min}$ \\
\hline \multirow{2}{*}{$\begin{array}{l}\text { An. gambiae } \\
\text { Kihihi }\end{array}$} & $4 \%$ DDT & 100 & 9 & 71 & 348 & 389 \\
\hline & Control & 50 & 2 & 0 & 0 & 0 \\
\hline \multirow{2}{*}{$\begin{array}{l}\text { An. gambiae } \\
\text { Katojo }\end{array}$} & $4 \% \mathrm{DDT}$ & 36 & 1 & 21 & 95 & 104 \\
\hline & Control & 10 & 0 & 0 & 0 & 0 \\
\hline
\end{tabular}


Table 5: Escape percentage response and mortality to DDT and LCT in Thailand.

\begin{tabular}{lcccc}
\hline & & & \multicolumn{2}{c}{$\%$ mortality } \\
\cline { 4 - 5 } Condition & Chem (No of tests) & No (\% escapes) & Exiled & Stayees \\
\hline Contact & DDT (85) & $78(92)$ & 0 & 42.8 \\
& LCT (77) & $72(94)$ & 1.4 & 100 \\
\hline \multirow{2}{*}{ Non-contact } & DDT (85) & $82(96)$ & 0 & 0 \\
& LCT (77) & $82(96)$ & 1.5 & 0 \\
\hline
\end{tabular}

Source: Adopted from Potikaskorri et al., 2005. Chem $=$ chemical.

\section{DISCUSSION}

Whereas a lot has changed politically and economically within the last 50 years, apparently the behavior of malaria main vectors in Uganda towards DDT has not changed. Notwithstanding that the tests used in 2000-2009 are greatly refined from their harbingers of 1957-1960 and despite the fact that the two study sets under consideration are not a replica of each other, still, it is noteworthy: that in both sets, DDT does not kill the mosquitoes instantly. The principle actions of DDT in malaria control are repellency, irritancy and slow but sure contact-killing. Whereas the 1957-1960 set recognized these properties of DDT as the chemical's greatest asset that could help Uganda get rid of malaria in a short time, the 2000-2009 studies consider these same properties as the greatest weakness of DDT. Clearly the latter studies favour the use of expensive organophophates and carbamates, new chemicals which are not widely researched; they seek to eliminate the timehonoured, residually effective, long-lasting and inexpensive DDT from malaria control. Whereas the 1957-1960 studies sought to eliminate malaria, the latter seem to encourage the opposite.

A bioassay test is an appraisal of the biological activity of a substance such as a chemical by testing its effect on an organism and comparing the result with some agreed standard. The roll back malaria (RBM) initiative defines the biological activity in IRS of malaria control as the killing of the vector and the standards are the knock-down insecticides for which the knock-down resistance (kdr) gene is still intact. So, their research emphasizes bioassays for vector resistance to knock-out insecticides, hence the name of their net work as the "African network on Vector Resistance to insecticide" (ANVR).

Science and society label any chemical used against insects as insecticide or insecticidal, meaning that the chemical is used to kill the insects (Grieco et al., 2007). Apparently, the work of ANVR is to use the chemicals that kill mosquitoes instantly to edge out those that control mosquitoes by other means. As shown in Table 2, in 1959 out of the 40 insects collected from non sprayed experimental huts in Katokye, only three died in 24 hours after exposure to 4\% DDT for 30 minutes. Similarly, in the same table, it is seen that in 2009 out of 100 insects exposed to $4 \%$ DDT for 60 minutes, only 54 died within 24 hours. Clearly in both cases DDT can not be regarded a "copper bullet" but rather a "rubber bullet", controlling the Anopheles gambiae mosquito not by instant killing but by dispersal. It may therefore be wrong to front DDT as an insecticide in malaria control (Achee et al., 2009; Grieco et al., 2007; Busvine, 1964).

In 1959-1960 studies in Kihihi De Zulueta et al. (1961) evaded discussing the bioassay results when they recommended DDT IRS and Chloroquine treatment for eradication of malaria from Uganda in a short time. On hind thought, this might explain why both DDT and chloroquine are simply endangered substances in Africa today. Malaria eradication from Africa is mere rhetoric: unattainable malaria vaccines are 
financially supported more than practical means (Shetty, 2009). In addition, contrary to the recent studies limited to mosquito susceptibility, the 1957-1960 did a lot of work on mosquito behaviour change in response to DDT and reported the changes in a highimpact journal, the East African Medical Journal (De Zulueta et al., 1961) but did not discuss them there, probably because the results tended to demote DDT if used literally as an insecticide. They were instead discussed in a low-impact Bulletin of WHO (Cullen and De Zulueta, 1962) with educative technical advice.

Nonetheless, the relatively slow knock out effect of DDT on Anopheles mosquitoes and its strong effects in malaria control observed in Kihihi were reported to WHO in 1962 (Cullen and De Zulueta, 1962). Since then, many researchers have drummed this change in behaviour of mosquitoes after exposure to DDT and to some extent pyrethroids (Busvine, 1964; Grieco et al., 2007). In particular, Busvine (1964) criticized the mosquito mortality as yard stick measure in malaria control, stressed the significance of DDT repellency with irritability tests and gave credit to the measurements of mosquito behaviour-change after exposure to control chemicals.

The immediate mosquito behaviour change reported by many scientists include excito-repellency so that mosquitoes caught in the house by spray seek immediate exit (Tables 2, 4 and 5) and those out side stop entering the house (De Zulueta et al., 1961); irritability so that the mosquitoes don't snug in sprayed environment but flee instead (Tables 5 and 6), and finally, a slow but sure kill with which, if for some reason the mosquito can not flee fast enough, it is demised by bits picked from the sprayed surface (Bimenya et al., 2009). This was very clearly stated to WHO in the last century particularly from the researches done in Uganda (De Zulueta et al., 1961; Cullen and De Zulueta, 1964). It was drummed by others in endless pleas (Busvine, 1964; Achee et al., 2009). Consequently, WHO gave a disclaimer to the bioassays such as currently used by ANVR that "interpretation of the resistance/susceptibility tests in malaria control is a responsibility of the researcher" (WHO R1186). For some reason, however, such disclaimers are printed in small fonts on protocols with WHO big font insignia.

The studies on Uganda mosquitoes presented in this work right from 1957-1960 to the 2000-2009 ANVR studies show that the Anopheles behaviouristic avoidance from DDT remains pronounced. This should be properly understood and well exploited because a higher degree of avoidance than normal (Figure 2) means a lower mosquito instant mortality but does not involve any increase in tolerance to the chemical (Eshgly, 1977) and while it is related to irritability, it is independent of repellency and toxicity (Achee et al., 2009).

Achee et al. (2009) categorized 14 chemicals historically used including DDT and pyrethroids and those currently under investigation for the first time such as carbamates and organophosphates for malaria vector control and found that chemicals exert different combinations of contact irritancy, spatial repellency and toxic actions. This was found true even within the same chemical classes. Spatial repellency and contact irritancy are different and independent from toxic actions. DDT and pyrethroids induce contact irritancy, spatial repellency and death so that despite the touted "resistance" (which is not easily developed in repelled species), irritancy and repellency will interrupt manmosquito contact to reduce malaria transmission. It is noteworthy that DDT and LCT share many properties including lack of non-contact toxicity (Table 5). This is indirectly illustrated by even the ANVR results (Table 1) and our results (Table 3).

The repellency and the lack of non contact toxicity properties of DDT (and LCT) are a double edged sword or a political glass described as half full/half empty: they were the properties mainly used by the 1957-1960 studies to make Kihihi habitable and to recommend DDT for malaria 
eradication/elimination/control by De Zulueta et al. (1961). They are the same properties that were used by the 2000-2009 studies to declare DDT and LCT inept because the two are unable to kill $98 \%$ - $100 \%$ of the mosquitoes, which is interpreted wrongly as a very low susceptible mark. These pesticides continue to kill Anopheles mosquitoes slowly but surely and over a long period at an affordable cost. This is where DDT excels; it effectively reduces person-mosquito contact in-doors, repels the malaria vector from the sprayed quarters, irritates the enemy into exile and kills the contacted enemy mosquito outside the sprayed quarters. DDT effectively does this over a long period (about a year) at an affordable price. These benefits of DDT and pyrethroids were not considered by the ANVR studies and yet their studies in a given locality for the test chemicals were a prerequisite recommended by WHO (2006.3).

\section{Conclusion}

The results of susceptibility/resistance bioassay studies alone on Anopheles species to DDT should not prevent the use of the chemical for malaria vector control in Uganda because the tests quantitatively measure only the quick kill action of the chemicals without due respect to their repellence, irritancy, and residual effects which are the classic strengths of DDT in malaria control and are still potent with malaria vector Anopheles gambiae in Uganda.

\section{Recommendation}

To overcome the malaria scourge in Uganda, DDT should be sprayed indoors concomitantly with household administration of effective antimalarials until a cost effective substitute to DDT is available.

\section{REFERENCES}

Achee NL, Sardell MR, Dusfour I, Chauhan KR, Grieco JP. 2009. Characterization of spatial repellent, contact irritant and toxicant chemical actions of standard vector control compounds. Journal of the Am. Mosq. Cont. Assoc., 25(2): 156-167.
ANVR. 2005. The work of the African Network on Vector Resistance to Insecticides 2000-2005 (Issued Nov. 2000).

Bimenya GS, Habarulema M, Okot JP, Olwa F, Okwi AL. 2010. Plasma levels of DDE/DDT and liver function in malaria control personne 16 months after indoor residual spraying with DDT in northern Uganda, 2008. SAMJ, 100(2): 118-121.

Busvine JR. 1964. The significance of DDTirritability tests on mosquitos. Bull. Wld. Hlth. Org., 31: 645-656.

Coluzzi A, Cullen JA, De Zulueta J. 1962. Afield trial in Uganda of a new method for determining the irritability of mosquitos to insecticides. WHO/Mal/345.

Cullen JR, De Zulueta J. 1962. Observation on the irritability of mosquitos to DDT in Uganda. Bull. Wld Hlth Org., 27: 239250.

Curtis CF. 2002. Should the use of DDT be revived for malaria vector control? Biomedica, 22: 455-61.

De Zulueta J, Kafuko GW, Cullen JR. 1961. The results of the first year of a malaria eradication pilot project in Northern Kigezi, Uganda. East African Medical Journal, 38(1): 1-26.

Grieco PJ, Achee LN, Chareonviriyaphap J, Suwonkard W, Chauhan K, Sardells MR, Roberts DR. 2007. A new classification system for the actions of IRS chemicals traditionally used for malaria control. Plos One, 2(8): 716.

Mazel HM. 2002. Bring back DDT and science (Editorial) $21^{\text {st }}$ Century Sciences and Technology Magazine (summer 2002), http//www.21stcentury sciencetech.com/outides/summer02/DDT. html.

Offoboche M. 2008 African Malaria Conference, Malaria eradication Initiative in Africa http://www. malariaeradicationinafrica.org/

Pautian T. 2000. The world wide malaria eradication effort. Science education 2000 http://www.bact.wise.edu:81/science Ed/discuss/msg Roaser S36. 
Potikaskorri J, Chareoniviriyophap, Bangs MJ, Prabaripai A. 2005. Behaviour responses to DDT and pyrthroids between Anopheles minimus species A, malaria vectors in Thailand. Am. J. Trop. Med. and Hyg., 73(2): 343-349.

Shetty Priya. 2009. Biomed analysis: To control or eradicate malaria http://www.scidev.net/en/health/opinions/ biomed_analysisto-control or-eradicate malaria.

Sollers-Riedel H. 1963. An international review of activities concerning mosquitoes in 1962. Proceedings of the $50^{\text {th }}$ Annual meeting of the New Jersey mosquito extermination association and the $19^{\text {th }}$ annual meeting of the American mosquito control association, 1963.

UNEP/POPS/DDTBP.1/2. 2008. Global status of DDT and its alternatives for use in vector control to prevent disease: stakeholders meeting to review the interim report for the establishment of a global partnership to develop alternatives to DDT. Geneva 3-5 Nov 2008.

WHO/CDS/NTD/WHOPES/GCDPP/2006.3

Guidelines for testing mosquito adulticides for indoor residual spraying and treatment of mosquito nets. 